\title{
Eine einfache Trennfunktion zur Gegenstromverteilung und ihre Bedeutung für die Praxis
}

\author{
Von E. Hecker \\ Aus dem Max-Planck-Institut für Biochemie, Tübingen \\ (Z. Naturforschg. 8 b, 77-86 [1953]; eingegangen am 10. Dezember. 1952) \\ Herrn Professor A. Buten andt zum 50. Geburtstage gewidmet
}

\begin{abstract}
Die Theorie der Gegenstromverteilung wird vervollständigt durch Ableitung einer Trennfunktion, die mit einem Minimum an Experimenten den günstigsten Weg und den zu erwartenden Erfolg einer Gegenstromverteilung vorausberechnen läßt. Für die Auswahl der Lösungsmittelsysteme und des günstigsten Volumenverhältnisses ergeben sich daraus wichtige Hinweise. Durch die Trennfunktion wird ein quantitativer Vergleich des Trenneffektes der Gegenstromverteilung mit dem anderer Trennverfahren möglich.
\end{abstract}

$\mathrm{D}_{\mathrm{s}}$ e Gegenstromverteilung ist 1926 zuerst von Jantzen ${ }^{1}$ zur Auftrennung von Basengemischen aus Teer erfolgreich eingesetzt und auch theoretisch behandelt worden. Jedoch erst zwei Jahrzehnte später hat sich die Methode im Laboratorium allgemein eingeführt, nachdem $\mathrm{Craig}^{2,3}$ ein einfaches, apparatives Prinzip zur Ausführung des Trennungsverfahrens angegeben hatte. Seither hat sich die Methode außerordentlich bewährt.

Im Bereich der organischen Chemie ist sie bei der Reindarstellung von Substanzen und zur verlustlosen Reinheitsprüfung mit Erfolg eingesetzt worden, während das Verfahren im Bereich der Biochemie sich hauptsächlich bei der schonenden Trennung von Naturstoffgemischen bewährt hat.

Insbesondere der Biochemiker muß an ein Trennungsverfahren sehr hohe Anforderungen stellen. Eine Methode wird um so wertvoller, je mehr die folgenden Bedingungen von ihr erfüllt werden:

1. Schonende Bearbeitung der Substanzgemische, nach Möglichkeit ohne Überschreiten der Zimmertemperatur.

2. Möglichkeit zur verlustlosen Trennung von kleinen und kleinsten Substanzmengen, wobei jedoch auch Bearbeitung von Substanzen im präparativen Maßstab möglich sein sollte.

3. Aus Gründen der Substanzersparnis sichere theoretische Grundlagen, so daß mit wenigen Ex-

1 E. J a ntz en, Dechema-Monographie Nr. 48, Bd. 5 , S. 100, Berlin 1932.

2 L. C. Cra i g, J. biol. Chemistry 155, 519 [1944].

3 L. C. Craig u. O. Post, Analyt. Chemistry 21, 500 [1949].

4 L. C. Craig, W. H a us mann, E. H. Ahrens u. E. J. H a rfenist, Analyt. Chemistry 23, 1326 [1951]. perimenten der Verlauf eines Trennungsganges möglichst weitgehend übersehen werden kann.

Die Gegenstromverteilung hat in diesen Punkten Verbesserungen erfahren, die ein schonendes Arbeiten auch bei der Entfernung der Lösungsmittel ermöglichen ${ }^{4,5}$ und die die Ausführung der Gegenstromverteilung als Mikromethode gestatten ${ }^{6}$. In der vorliegenden Arbeit soll die Theorie der Gegenstromverteilung durch Ableitung einer Trennfunktion vervollständigt werden.

Der Hauptwert der folgenden Ableitungen liegt darin, daß sie gestatten, unter Einsparung wertvoller Substanzen die besten Versuchsbedingungen aufzusuchen, die Grenzen der Möglichkeiten zu erkennen und die Wirksamkeit verschiedener Trennungsverfahren zahlenmäßig zu vergleichen.

$$
\begin{gathered}
\text { Bedeutung und Ermittlung } \\
\text { des Trennfaktors }
\end{gathered}
$$

Die Gegenstromverteilung besteht aus einer Aufeinanderfolge zahlreicher Einzelextraktionen, die im Idealfalle dem Nernstschen Verteilungssatz gehorchen ${ }^{*}$.

Der N e rn st sche Verteilungssatz besagt, daß sich eine Substanz zwischen zwei beschränkt mischbaren Phasen unabhängig von der Konzentration und der

5 E. Hecker u. P. K a r ls on, Chem. Ing. Techn.. im Druck.

6 E. H e c k e r, Chem. Ing. Techn., im Druck.

* Die folgenden Ableitungen sind alle unter der Voraussetzung der Gültigkeit des Nerns tschen Verteilungssatzes gemacht. Ưber den Gültigkeitsbereich vgl. 7 .

$7 \mathrm{P}$. K arls on u. E. H e c k e r, Z. Naturforschg. $5 \mathbf{b}$. 237 [1950]. 
Gegenwart von Begleitstoffen in einem konstanten und reproduzierbaren Verhältnis verteilt. Jeder einzelnen Substanz eines Gemisches kann also ein für

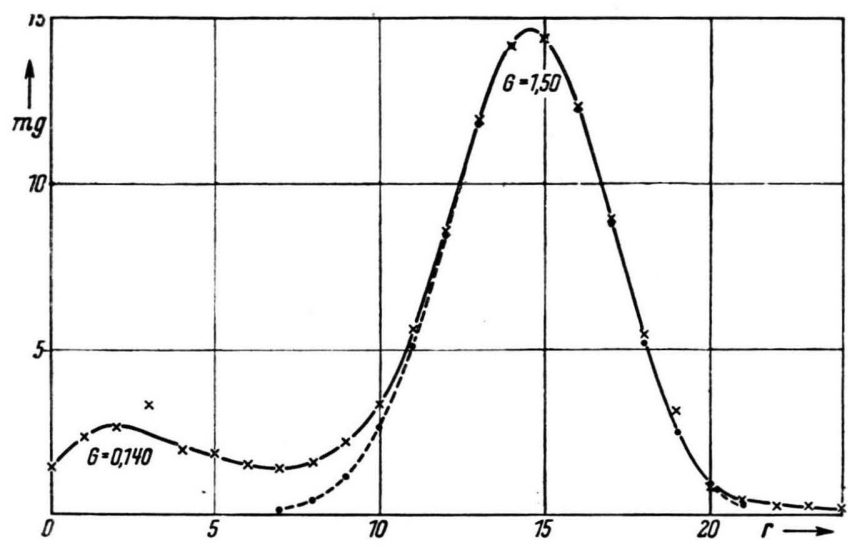

Abb. 1. Trennung eines Triterpens $(G=1,50)$ von einer Verunreinigung $(G=0,140) ; \beta=10,7 ;(-x-)$ gefunden, $(--\cdot-)$ berechnet. System Benzin (Sdp. 150-160 $) / \mathrm{Me}-$ thanol; $V=0,73^{*}$.

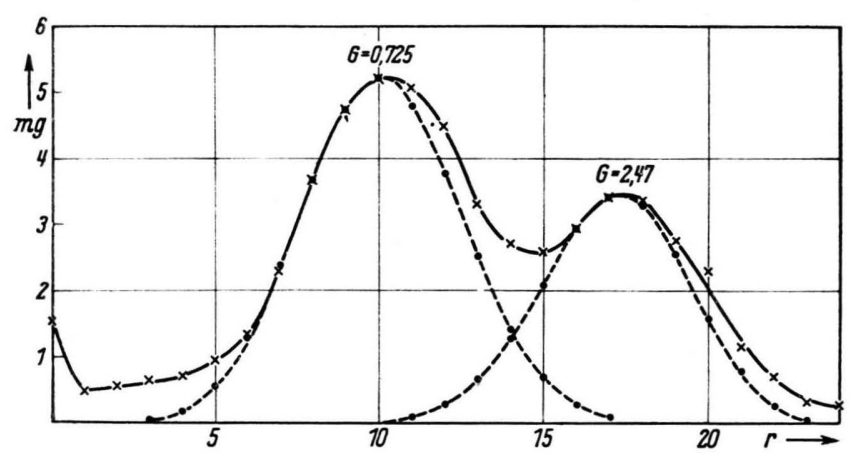

Abb. 2. Verteilung des Reaktionsproduktes aus einer Reduktion nach M e e rwe in-P onnd orf im System 70-proz. wäßr. Methanol $/ \mathrm{CCl}_{4}-\mathrm{CHCl}_{3}(3: 2), V=1 . G=0,725$ 16-Isonitroso-13-iso-androstan-ol-(3 $\beta)$-on-(17), $G=2,74$ 16-Isonitroso-13-iso-androstan-diol- $(3 \beta, 17)$; (-x-) gefunden, (----) berechnet.

sie charakteristischer Verteilungskoeffizient $K$ zugeschrieben werden, der durch

$$
\frac{c_{1}}{c_{0}}=K
$$

definiert ist, wobei $c_{1}$ die Konzentration in der oberen Phase, $c_{2}$ in der unteren Phase bedeuten.

* Für die Überlassung der experimentellen Daten (Abb. 1 u. Abb. 2) bin ich den Herren Dipl.-Chem. H. T e ufel und Dipl.-Chem. W. F ru s t orfer zu Dank verpflichtet.
Bei der praktischen Ausführung der Gegenstromverteilung spielt das Volumenverhältnis $V=V_{p} / V_{q}$ der beiden Phasen eine bedeutende Rolle. Es ist daher zweckmäßig, statt des klassischen VerteilungsKoeffizienten $K$ die Verteilungs-Zahl $G$ einzuführen. Sie ist definiert durch

$$
\frac{p}{q} \equiv G=K \cdot V
$$

wenn $p$ den relativen Anteil der Substanzmenge in der Oberphase, $q$ in der Unterphase bedeuten. Es ist also $p+q=1$. Für den Spezialfall $V=1$ ist $G=K$. Wird die Verteilungszahl zur Charakterisierung einer Substanz benützt, so muß gleichzeitig auch das Volumenverhältnis, bei dem $G$ gewonnen wurde, angegeben werden. Man kann aber auch mit Gl. (2) auf $K$ umrechnen.

Ein quantitatives Maß für die Trennbarkeit zweier Substanzen A und B durch Gegenstromverteilung ist das Verhältnis ihrer Verteilungszahlen $G_{\mathrm{A}}$ und $G_{\mathrm{B}}$, der Trennfaktor $\beta$. Er ist von $\mathrm{V}$ a r te re s s i a n und Fens ke ${ }^{8}$ eingeführt worden. Es ist

$$
\beta=\frac{G_{\mathrm{A}}}{G_{\mathrm{B}}}=\frac{K_{\mathrm{A}}}{K_{\mathrm{B}}} \geqq 1 .
$$

Für $\beta=1$ ist eine Trennung der Substanzen unmöglich. Bei $\beta>10$ lassen sich zwei Substanzen nach dem Grundprozeß mit wenigen Verteilungsschritten (n) quantitativ trennen, wie man aus Abb. 1 erkennt. Mit der gleichen Anzahl von Verteilungsschritten lassen sich aber zwei Substanzen mit einem Trennfaktor von $\beta=3,41$ nicht mehr quantitativ trennen (Abb. 2), und es ist die Frage nach einer quantitativen Beziehung zwischen $\beta$ und $n$ zu stellen. Eine solche Trennfunktion $n=f(\beta)$ würde bei Kenntnis der Verteilungszahlen zweier Substanzen sofort darüber entscheiden lassen, ob das gestellte Trennungsproblem mit der Zahl der zur Verfügung stehenden Verteilungselemente und einem vorgegebenen Lösungsmittelsystem zu lösen ist bzw. wieviel Verteilungselemente dazu mindestens erforderlich sind.

Für den Grundprozeß ist durch die Zahl $n$ der Verteilungsschritte die Zahl $z$ der dazu notwendigen Verteilungselemente in einfacher Weise bestimmt. Es ist $n=z-1^{7}$. Bei den Entnahmeverfahren hängt $n$ außer von $z$ auch von der Anzahl der entnommenen Phasen ab. Auch für diese Verfahren lassen sich Trennfunktionen ableiten. Sie bringen jedoch keinen wesentlichen Vorteil, da der Trenneffekt bei diesen Verfahren höchstens den

8 K. A. Varteressian u. M. R. Fenske. Ind. Engng. Chem. 29, 270 [1937]. 
eines Grundprozesses mit gleicher Zahl von Verteilungsschritten erreicht. Meist ist die Trennwirkung aber schlechter ${ }^{7}$. Die für den Grundprozeß gültige Trennfunktion gibt deshalb in jedem Fall das Minimum der zur Trennung notwendigen Verteilungsschritte an.

Die Ermittlung des Trennfaktors zweier Substanzen ist einfach, wenn beide in reiner Form vorliegen. Weit häufiger kommen jedoch Gemische unbekannter Substanzen vor. Die Trennfaktoren der Komponenten solcher Mischungen sind nur durch eine orientierende Gegenstromverteilung zu ermitteln. Am besten zieht man dazu den Grundprozeß heran.

Um eine solche erste Verteilung rasch und sicher ausführen zu können, ist die Kenntnis der Verteilungszahl des Gemisches

$$
\frac{p_{\mathrm{M}}}{q_{\mathrm{M}}}=G_{\mathrm{M}}
$$

von Nutzen. Man bestimmt sie durch Verteilung einer geringen Menge des Gemisches in einem kleinen Scheidetrichter bei einem Volumenverhältnis von $V=1$. Hierbei haben sich in unserem Institut kleine zylindrische Scheidetrichter $(15 \times 1,5 \mathrm{~cm})$ bewährt. Liegt die Verteilungszahl in dem benützten Lösungsmittelsystem nicht zwischen 0,5 und $2,0^{*}$, so muß man ein anderes Lösungsmittelpaar aufsuchen, in dem die Verteilungszahl zwischen den angegebenen Werten liegt. Für welchen Wert man sich entscheidet, hängt davon $\mathrm{ab}$, welche Substanzmengen mit $G<1$ und $G>1$ zu erwarten sind. C raig 9 hat die Meinung vertreten, daß der Verteilungskoeffizient des Substanzgemisches nach Möglichkeit $=1$ sein sollte, um optimale Trennung der Substanzen zu erhalten. Dies trifft jedoch nur zu, wenn gleiche Mengen der Komponenten des Gemisches vorliegen. Bei Gemischen unbekannter Zusammensetzung wird man daher nicht zu viel Zeit darauf verwenden, $G_{\mathrm{M}}$ exakt gleich eins zu machen.

Aus der Lage der Maxima der orientierenden Grundverteilung, die u. U. geschätzt werden müssen, kann man mit Hilfe der Gl. (5) die jeweilige Verteilungszahl berechnen.

$$
\begin{aligned}
G & =\frac{r_{\mathrm{m}}+0,5}{n-r_{\mathrm{m}}+0,5}, \\
r_{\mathrm{m}} & =\frac{(n+1) G}{1+G}-0,5 ;
\end{aligned}
$$

$G$ ist die Verteilungszahl, $n$ die Zahl der Verteilungsschritte und $r_{\mathrm{m}}$ die Fraktionsnummer des Maxi-

\footnotetext{
* Diese Werte hängen lediglich von der Dimension der Verteilungsapparate ab. Die meisten Geräte sind so gebaut, daß man das Volumenverhältnis bis $V=2,0$ variieren kann. Unter einen Wert von $V=0,5$ zu gehen, ist in den meisten Fällen nicht ratsam.

9 L. C. Craig, Fortschr. chem. Forschg. 1, 294 [1949].
}

mums der Kurve, die nicht ganzzahlig zu sein braucht. Für $n=24$ ergibt sich z. B. bei $r_{\mathrm{m}}=14,3$ ein $G=1,45$ und für $G=0,5$ ein $r_{\mathrm{m}}=7,84$. Die Gln. (5) und (6) gelten exakt im Bereich $0,15 \leqq G$ $\leqq 6,1$ oder, anders ausgedrückt, für Maxima, die zwischen $r=n / 8$ und $r=n-n / 8$ liegen. Gl. (6) ist für die Vorausberechnung der Lage eines Maximums oft wichtig. Sie geht bei Vernachlässigung der kleinen Glieder bei großen $n$ in die von $\mathrm{Cr}$ a ig ${ }^{2}$ und B o ck ${ }^{10}$ gegebene Näherungsgleichung (6a) über:

$$
r_{\mathrm{m}}=\frac{n \cdot G}{1+G}=n \cdot p .
$$

Ableitung der allgemeinen Trennfunktion

Eine Beziehung zwischen den Verteilungskoeffizienten bzw. -zahlen zweier Substanzen und der Zahl der zu ihrer quantitativen Trennung notwendigen Verteilungsschritte ist von $\mathrm{Zilch}$ und $\mathrm{Dut}$ to ${ }^{11}$ zitiert worden, jedoch liefert diese Gleichung in der Interpretation der Autoren viel zu niedrige $n$-Werte, auch wird der Einfluß des Volumenverhältnisses auf die Zahl der Verteilungsschritte nicht berücksichtigt, und auch bei $\mathrm{Nichols}{ }^{12}$ vermißt man eine einfache und geschlossene Darstellungsweise. Von $\mathrm{Craig}{ }^{13}$ wurde eine graphische Lösung des Problems angegeben, wobei die Formeln aus der Theorie der Destillation entliehen sind. Das Verfahren ist sehr umständlich und nur bei großen Trennfaktoren praktisch durchführbar. Die Praxis verlangt jedoch eine einfache Formel, die leicht zu übersehen ist, und die bei graphischer Darstellung möglichst viele Einzelprobleme zu erfassen gestattet.

Eine Beziehung, die die gestellten Anforderungen erfüllt, läßt sich ableiten, wenn man die Gegenstromverteilung als statistisches Problem darstellt. Die Einführung der Verteilungszahl an Stelle des Verteilungskoeffizienten ergibt sich aus dem Ansatz von selbst.

In statistischer Auffassung gibt eine Verteilungskurve die Wahrscheinlichkeit an, mit der die Moleküle einer Substanz nach $n$ Verteilungsschritten in den Fraktionen $r=0$ bis $r=n$ auftreten. Für das Maximum der Kurve ist diese Wahrscheinlichkeit am

10 R. M. B o ck, J. Amer. chem. Soc. 72, 4269 [1950]. 11 K. T. Zilch u. H. J. Dutton, Analyt. Chemistry 23, 775 [1951].

12 P. L. Nichols, Analyt. Chemistry 22, 915 [1950].

13 L. C. Craig u. D. C raig. Technique of Org. Chemistry III, New York 1950. 
größten, und man kann aus der Lage des Maximums jeder Substanz mittels Gl. (5) eine Verteilungszahl $G$ zuordnen, die für die Substanz bei gegebenem Phasenpaar und Volumenverhältnis charakteristisch ist. In dieser Auffassung kann man die zu jeder Verteilungszahl $G$ gehörige Verteilungskurve anstatt durch die Ausbreitung des Binoms $(p+q)^{n}$ durch eine $\mathrm{G}$ a u $B$ sche Glockenkurve darstellen. Bei genügend

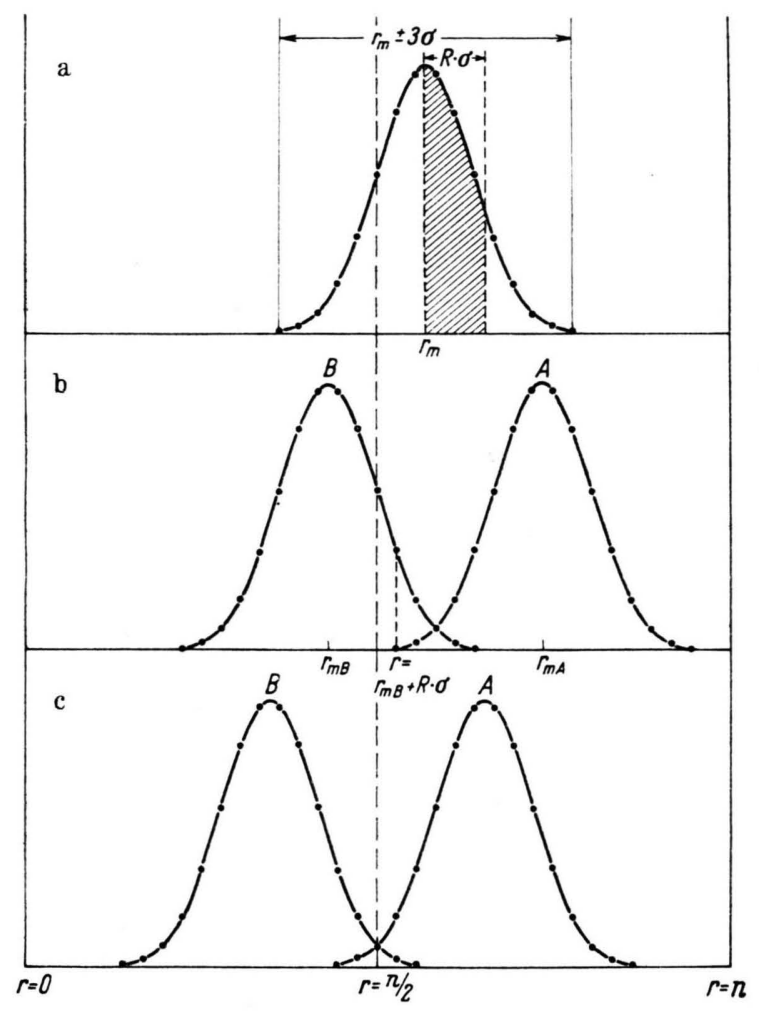

Abb. 3.

großen Werten von $n(n \geqq 25)$ gilt dann für die relative Substanzmenge in den Fraktionen:

$$
T_{n, x}=\frac{1}{\sqrt{2 \pi n p q}} e^{\frac{x^{2}}{2 n p q}},
$$

wobei sich die Fraktionsnummern $x$ auf das Maximum der Kurve als Nullpunkt beziehen ${ }^{13}$. Bezogen auf ein Koordinatensystem mit der Abszisse von $r=0$ bis $r=n$ lautet Gl. (7):

$$
T_{n, r}=\frac{1}{\sqrt{2 \pi n p q}} e^{\frac{\left(r_{\mathrm{m}}-r\right)^{2}}{2 n p q}}
$$

\begin{tabular}{|c|c|}
\hline $\begin{array}{c}\% \text { reine } \\
\text { Substanz }\end{array}$ & $R$ \\
\hline 50 & 0,0000 \\
60 & 0,2530 \\
70 & 0,5245 \\
80 & 0,8415 \\
90 & 1,2815 \\
95 & 1,6448 \\
97,5 & 1,9600 \\
99,0 & 2,5750 \\
99,73 & 3,0000 \\
\hline
\end{tabular}

Tab. 1.

$r_{\mathrm{m}}=$ Fraktionsnummer des Maximums. Gl. (8) gilt mit genügender Näherung für $0,2 \leqq G \leqq 5,0$ u. $n \geqq 25$.

Sollen nun zwei Substanzen A und B mit den Verteilungszahlen $G_{A}$ und $G_{B}$ praktisch quantitativ getrennt werden, so müssen auf das Gemisch offenbar so viele Verteilungsschritte angewandt werden, daß sich die beiden Verteilungskurven bei einem Ordinatenwert schneiden, der sehr viel kleiner ist als der der Maxima. Ein Ansatz, der auf diesen Grundgedanken zurückgeht, führte zu einer sehr unhandlichen Trennfunktion ${ }^{14}$ und ist schon mehrfach durchgerechnet worden ${ }^{12,15}$.

Das gestellte Problem wird jedoch wesentlich vereinfacht, wenn man als Maß für den Grad der Trennung zweier Substanzen die Fläche unter den Verteilungskurven benützt. $\mathrm{Zu}$ diesem Zweck ist die in der Statistik übliche Streuung oder Standardabweichung $\sigma$ von Nutzen. In den bekannten Größen ausgedrückt, ist

$$
\sigma=\ln p q .
$$

Es besteht ganz allgemein eine Beziehung zwischen der Prozentzahl der Moleküle, die in den Bereich zwischen $r=r_{\mathrm{m}}-3 \sigma$ und $r_{\mathrm{m}}+R \cdot \sigma$ unter die Kurve fallen (Abb. 3 a). Die Zahlenwerte von $R$ und die zugehörigen Prozentzahlen können aus Tab. 1 entnommen werden ${ }^{\mathbf{1 6}}$.

Führt man also die Verteilung zweier Substanzen A und $\mathrm{B}$ so weit, daß die Fraktion $r=r_{\mathrm{mA}}-3 \sigma$ mit der Fraktion $r=r_{\mathrm{mB}}+R \cdot \sigma$ zusammenfällt, so ist die Substanz B bis zur Fraktion $r=r_{\mathrm{mB}}+R \cdot \sigma$ praktisch als rein anzusehen (Abb. $3 \mathrm{~b}$ ). Wir bezeichnen den Faktor $R$ deshalb als Reinheitsfaktor. Man kann also ansetzen

14 E. H e c k e r, Diplomarbeit Tübingen 1950.

15 A. J. P. M a rtin u. R. L. M. S ynge. Biochem. J. 35, 1358 [1941].

16 S. K o ll e r, Graphische Tafeln z. Beurteilung statistischer Zahlen. Dresden u. Leipzig 1943. 


$$
r_{\mathrm{mA}}-3 \sigma_{\mathrm{A}}=r_{\mathrm{mB}}+R \sigma_{\mathrm{B}},
$$

und mit Gl. (9) ergibt sich

$$
r_{\mathrm{mA}}-r_{\mathrm{mB}}=R \sqrt{n p_{\mathrm{B}} q_{\mathrm{B}}}+3 \sqrt{n p_{\mathrm{A}}} q_{\mathrm{A}}
$$

und daraus mit Gl. (6a)

$$
n\left(p_{\mathrm{A}}-p_{\mathrm{B}}\right)=R \sqrt{n p_{\mathrm{B}} q_{\mathrm{B}}}+3 \sqrt{n p_{\mathrm{A}} q_{\mathrm{A}}} .
$$

Diese Gleichung läßt sich nun noch umformen, wenn man $p=G / 1+G, q=1 / 1+G$ und $G_{\mathrm{A}} / G_{\mathrm{B}}=\beta$ setzt und anstatt $G_{\mathrm{A}} \cdot G_{\mathrm{B}}$ zur Abkürzung $\alpha$ einführt. Für Gl. (12) ergibt sich dann

$$
\begin{array}{r}
n=\frac{\beta}{(\beta-1)^{2}}\left[9\left(2+\frac{a+\beta}{\sqrt{\alpha \beta}}\right)+6 R\left(\frac{a+1}{\sqrt{a}}+\frac{\beta+1}{\sqrt{\beta}}\right)\right. \\
\left.+R^{2}\left(2+\frac{a \beta+1}{\sqrt{\alpha \beta}}\right)\right] .
\end{array}
$$

Dies ist die gesuchte Trennfunktion. Sie gilt mit genügender Näherung für $0,2 \leqq G \leqq 5,0$ und $n \geqq 25$. Die Gleichung ist jedoch für den praktischen Gebrauch sehr umständlich. Man erkennt, daß die Zahl der Verteilungsschritte außer vom Trennfaktor $\beta$ auch vom Produkt $\alpha$ der Verteilungszahlen abhängt. Die vollständige Trennfunktion ist also eine Funktion mit drei unabhängigen Veränderlichen $n=f(\alpha, \beta, R)$. Die Größe $\alpha$ bedarf jedoch noch näherer Erläuterung.

\section{Der Volumenfaktor und seine Bedeutung}

In das Produkt der Verteilungszahlen geht, im Gegensatz zum Trennfaktor, das Volumenverhältnis der beiden Phasen ein. Wir bezeichnen $\alpha$ deshalb als den Volumenfaktor. Es ist

$$
a=G_{\mathrm{A}} G_{\mathrm{B}}=K_{\mathrm{A}} K_{\mathrm{B}} V^{2} .
$$

Wählt man das Volumenverhältnis $V$ so, daß $\alpha=1$ wird, nämlich

$$
V=\frac{1}{\sqrt{K_{\mathrm{A}}} K_{\mathrm{B}}}{ }^{*}
$$

so geht Gl. (14) über in $G_{\mathrm{A}}=1 / G_{\mathrm{B}}$, d. h. die Verteilungskurven der Substanzen $\mathrm{A}$ und $\mathrm{B}$ liegen nach einer $n$-fachen Verteilung symmetrisch zu $r=n / 2$ (Abb. 3c).

Dieser Sonderfall hat sehr große praktische Bedeutung, denn bei $\alpha=1$ wird für Gl. (13) $(\partial n / \partial \alpha)_{\beta=\text { const. }}=0$,

* Diese Beziehung haben bereits Bush und Densen ${ }^{17}$ sowie $\mathrm{S}$ t e $\mathrm{n} \mathrm{e}^{18}$ auf andere Weise abgeleitet.

17 M. T. Bus h u. P. M. D en s e n, Analyt. Chemistry 20, 121 [1948].

\begin{tabular}{|l|l|l|l|l|}
\hline$G_{\mathrm{B}}$ & $G_{\mathrm{A}}$ & $\beta$ & $\alpha$ & $n$ \\
\hline 0,2 & 0,8 & 4,0 & 0,16 & 88 \\
0,5 & 2,0 & 4,0 & 1,00 & 72 \\
1,25 & 5,0 & 4,0 & 6,25 & 88 \\
0,5 & 1,0 & 2,0 & 0,5 & 306 \\
0,707 & 1,414 & 2,0 & 1,0 & 292 \\
1,0 & 2,0 & 2,0 & 2,0 & 306 \\
\hline
\end{tabular}

Tab. 2.

d. h. es ist für diesen Fall ein Minimum an Verteilungsschritten zur Auftrennung der Substanzen erforderlich. Dies wird in Tab. 2 an einem Zahlenbeispiel veranschaulicht, das für quantitative Trennung $(R=3)$ berechnet ist. Die Tabelle zeigt, daß für $\alpha=1$ ein Minimum an Verteilungsschritten zur quantitativen Trennung benötigt wird.

Die beiden Beispiele wurden für reziproke $\alpha$-Werte berechnet. Man erkennt aus dem Vergleich der Werte für $\beta=4,0$ und $\beta=2,0$, da $\beta$ die Volumenkorrektur einen um so größeren, prozentualen Gewinn an Verteilungsschritten bringt, je stärker sich der Volumenfaktor von 1 unterscheidet.

Man hat es also in der Hand, durch Anpassung des Volumenverhältnisses $V$ an die Verteilungskoeffizienten der zu trennenden Substanzen im Sinne der Gl. (14a) die Zahl der Verteilungsschritte zu reduzieren.

\section{Die vereinfachte Trennfunktion}

Der für die Praxis bedeutsame Sonderfall $\alpha=1$ ergibt für die allgemein gültige Trennfunktion eine weitgehende Vereinfachung.

Für $\alpha=1$ wird aus Gl. (13)

$$
n=\frac{(R+3)^{2} \beta(2+V / \beta+1 / \sqrt{\beta})}{(\beta-1)^{2}}
$$

und in anderer Form

$$
n=\frac{(R+3)^{2} \sqrt{\beta}}{(\beta-1)^{2}}(\sqrt{\beta}+1)^{2} ;
$$

zuletzt erhält man

$$
n=\frac{(R+3)^{2}}{\frac{\beta+1}{\sqrt{\beta}}-2}
$$

* E. H e cker, Diss. Tübingen 1952.

18 S. S t e ne, Ark. Kem. Mineral. Geol., Ser. A 18. Nr. 18, 1 [1944]. 
Dies ist die Trennfunktion für den praktisch wichtigsten Sonderfall $\alpha=1$.

Mit Gl. (17) und Tab. 1 kann man die Zahl der Verteilungsschritte sofort angeben, die notwendig ist. um zwei Substanzen in einem bestimmten Reinheitsgrad zu erhalten. Will man z. B. praktisch 100-proz.

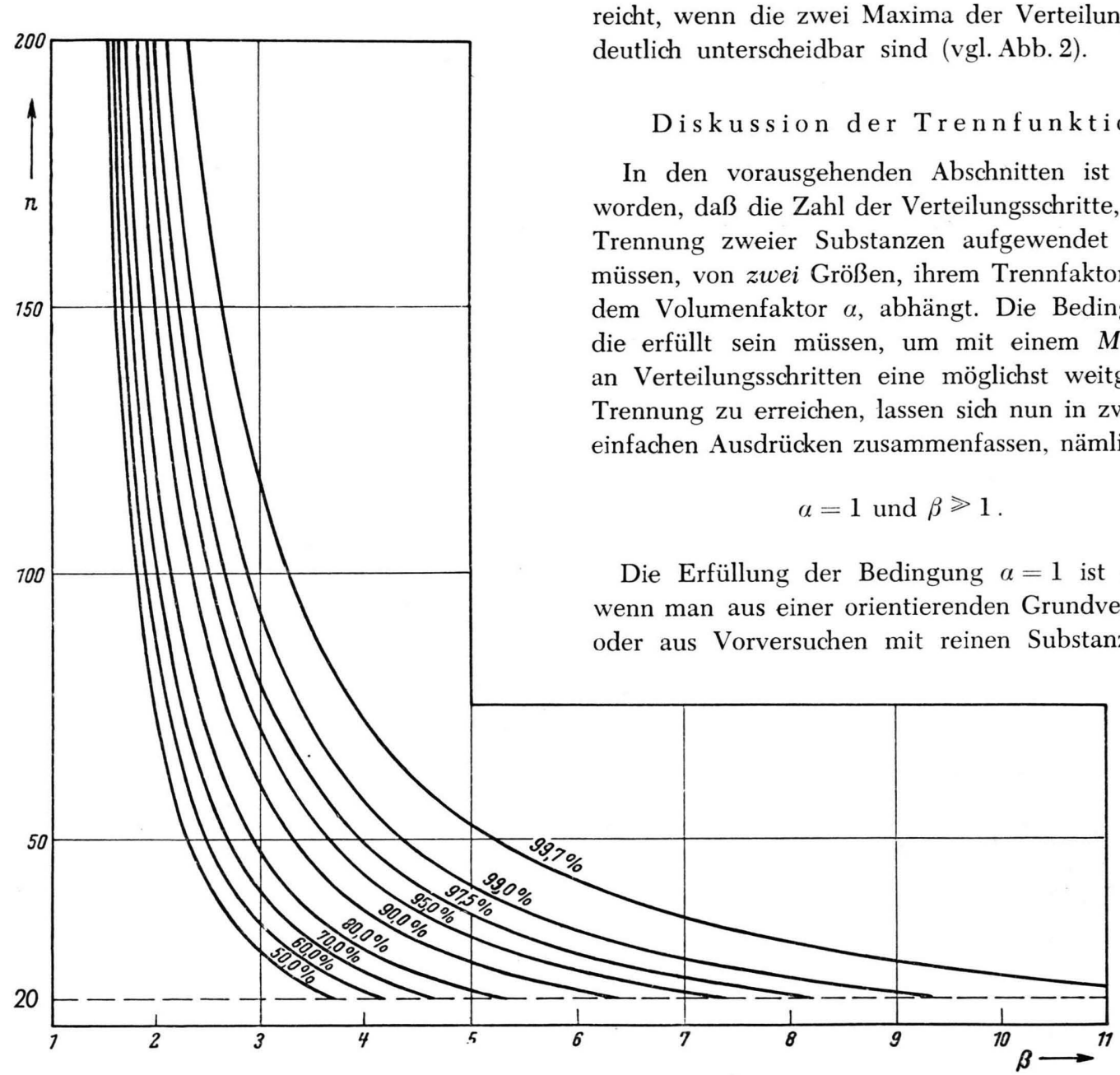

Abb. 4. Die Trennfunktion für den Sonderfall $\alpha=1$ in graphischer Darstellung.
Aus Tab. 3 läßt sich als Faustregel ableiten: Hat man zwei Substanzen A und B soweit getrennt, daß sie in 50-proz. Reinheit vorliegen, so braucht man zur Gewinnung 100-proz. reiner Substanz das 4-fache an Verteilungsschritten. Der Zustand einer 50-proz. Trennung der beiden Substanzen ist etwa dann erreicht, wenn die zwei Maxima der Verteilungskurve deutlich unterscheidbar sind (vgl. Abb. 2).

In den vorausgehenden Abschnitten ist gezeigt worden, daß die Zahl der Verteilungsschritte, die zur Trennung zweier Substanzen aufgewendet werden müssen, von zwei Größen, ihrem Trennfaktor $\beta$ und dem Volumenfaktor $\alpha$, abhängt. Die Bedingungen, die erfüllt sein müssen, um mit einem Minimum an Verteilungsschritten eine möglichst weitgehende Trennung zu erreichen, lassen sich nun in zwei sehr einfachen Ausdrücken zusammenfassen, nämlich

$$
\alpha=1 \text { und } \beta \gg 1 \text {. }
$$

Die Erfüllung der Bedingung $\alpha=1$ ist einfach, wenn man aus einer orientierenden Grundverteilung oder aus Vorversuchen mit reinen Substanzen die reine Substanzen isolieren, so setzt man $R=3$ und erhält dann für jedes beliebige $\beta$ die dazu notwendige Zahl von Verteilungsschritten unter der Voraussetzung, daß das Volumenverhältnis nach Gl. (14 a) den Verteilungskoeffizienten angepaßt wird.

Die für die Reinheitsgrade der Tab. 1 nach Gl. (17) für Trennfaktoren zwischen $\beta=1,5$ und 11 berechneten Verteilungsschritte sind in Tab. 3 angegeben und in Abb. 4 graphisch dargestellt. Diese $n$-Werte gelten mit genügender Genauigkeit, wenn $n \geqq 25$ ist, und sind um so genauer, je kleiner $\beta$ ist.
Verteilungskoeffizienten kennt. Man braucht dann nur nach Gl. (14a) das Volumenverhältnis für $\alpha=1$ auszurechnen. Nach beendeter Verteilung liegen dann die Maxima der Verteilungskurven symmetrisch zu $r=n / 2$. Wie man aus Tab. 2 erkennt, kann die Volumenanpassung eine Reduzierung der Zahl der Verteilungsschritte um 20\% ausmachen.

Von noch größerem Einfluß auf die Anzahl der Verteilungsschritte ist der Trennfaktor $\beta$. Aus Abb. 4 erkennt man, daß die Zahl der Verteilungsschritte mit kleiner werdendem $\beta$ und bei größeren Anforde- 


\begin{tabular}{|c|c|c|c|c|c|c|c|c|c|}
\hline$\beta$ & $n_{99,73 \%}$ & $n_{99 \%}$ & $n_{97,5^{\circ} \%}$ & $n_{95 \%}$ & $n_{90 \%}$ & $n_{80 \%}$ & $n_{70 \%}$ & $n_{60 \%}$ & $n_{50 \%}$ \\
\hline 11,0 & 22 & & & & & & & & \\
\hline 10,0 & 24 & & & & & & & & \\
\hline 9,0 & 27 & 21 & & & & & & & \\
\hline 8,0 & 30 & 24 & 21 & & & & & & \\
\hline 7,0 & 35 & 28 & 24 & 21 & & & & & \\
\hline 6,0 & 42 & 33 & 28 & 25 & 21 & & & & \\
\hline 5,0 & 53 & 42 & 36 & 32 & 27 & 22 & & & \\
\hline 4,5 & 61 & 48 & 41 & 36 & 31 & 25 & 21 & & \\
\hline 4,0 & 72 & 57 & 49 & 43 & 37 & 29 & 25 & 21 & \\
\hline 3,5 & 89 & 70 & 60 & 53 & 45 & 36 & 31 & 26 & 22 \\
\hline 3,0 & 116 & 92 & 79 & 70 & 59 & 48 & 40 & 34 & 29 \\
\hline 2,7 & 143 & 113 & 97 & 86 & 73 & 59 & 49 & 42 & 36 \\
\hline 2,4 & 185 & 146 & 126 & 111 & 94 & 76 & 64 & 55 & 46 \\
\hline 2,2 & 229 & 181 & 155 & 137 & 117 & 94 & 79 & 67 & 57 \\
\hline 2,0 & 292 & 230 & 198 & 175 & 149 & 120 & 101 & 86 & 72 \\
\hline 1,9 & 346 & 274 & 236 & 208 & 177 & 142 & 119 & 102 & 87 \\
\hline 1,8 & 413 & 326 & 281 & 248 & 211 & 169 & 143 & 121 & 103 \\
\hline 1,7 & 508 & 401 & 345 & 305 & 259 & 208 & 175 & 149 & 127 \\
\hline 1,6 & 649 & 512 & 441 & 389 & 331 & 266 & 224 & 191 & 162 \\
\hline 1,5 & 872 & 689 & 593 & 523 & 445 & 357 & 301 & 256 & 218 \\
\hline
\end{tabular}

Tab. 3.

rungen an die Reinheit der Substanzen sehr rasch zunimmt. So können z. B. zwei Substanzen mit $\beta=4,0$ durch eine Apparatur mit 72 Verteilungselementen $(n \approx z)$ nach dem Grundprozeß quantitativ getrennt werden. Bei einem Trennfaktor von $\beta=2,0$ sind dazu schon 292 Verteilungselemente notwendig, oder man erhält mit 72 Verteilungselementen nur zu $50 \%$ reine Substanzen (Tab. 3). Wenn man also nicht sehr große und kostspielige Apparaturen aufstellen will, muß man dafür Sorge tragen, daß der Trennfaktor möglichst gro $\beta$ ist. Dabei wirken sich bei kleineren Trennfaktoren bereits geringfügige Vergrößerungen

\begin{tabular}{|l|c|c|c|c|c|}
\hline & $\begin{array}{c}\text { Methanol- } \\
\text { Wasser } \\
50: x\end{array}$ & $G_{\mathrm{A}}$ & $G_{\mathrm{B}}$ & $\beta$ & $n$ \\
\hline$n$-Heptan- & $50: 0$ & 1,227 & 0,643 & 1,98 & 305 \\
$\begin{array}{l}\text { Benzol } \\
(10: 1)\end{array}$ & $50: 1$ & 1,86 & 0,849 & 2,19 & 231 \\
& $50: 2$ & 2,74 & 1,006 & 2,72 & 141 \\
$n$-Heptan- & $50: 0$ & 1,256 & 0,607 & 2,07 & 269 \\
$\begin{array}{l}\text { Cyclohexan-Benzol } \\
(10: 10: 2)\end{array}$ & $50: 1$ & 1,901 & 0,815 & 2,33 & 198 \\
$n$-Heptan- & $50: 2$ & 2,67 & 1,005 & 2,66 & 147 \\
$\begin{array}{l}\text { Benzol } \\
(10: 2)\end{array}$ & $50: 0$ & 1,17 & 0,752 & 1,55 & 600 \\
\hline $50: 1$ & 1,493 & 0,865 & 1,725 & 481 \\
\hline
\end{tabular}

Alle Verteilungszahlen wurden mit $V=1$ bestimmt. $G_{\mathrm{A}}=$ Anthrachinoncarbonsäureester des $n$-Octanols; $G_{\mathrm{B}}=$ Anthrachinoncarbonsäureester des Hexen-(2)-ol-(1); $n=$ Zahl der Verteilungsschritte für $a=1$ nach Abb. 4 bzw. Gl. (17).

Tab. 4 . von $\beta$ in einer wesentlichen Verringerung der Zahl der Verteilungsschritte aus (Tab. 4).

Die Größe des Trennfaktors kann durch passende Auswahl des Lösungsmittelsystems beeinflußt werden, und besonders dann, wenn nur kleinere Verteilungsapparaturen $(z<100)$ zur Verfügung stehen, muß man für einen möglichst großen. Trennfaktor Sorge tragen und auf die Auswahl des Lösungsmittelsystems größte Sorgfalt verwenden. Bereits $\mathrm{J}$ a $\mathrm{tz} \mathrm{e}^{1}{ }^{1}$ hat darauf hingewiesen, daß der Trennfaktor zweier Substanzen um so größer ist, je weniger sich die beiden Phasen des Lösungsmittelsystems miteinander mischen. Dies wird durch ein Beispiel in Tab. 4 veranschaulicht.

Um ein geeignetes Lösungsmittelsystem zur Verteilung von Anthrachinon-carbonsäureestern zu ermitteln, wurden zwei der Ester in Lösungsmittelsystemen verteilt, deren Oberphase aus einem Kohlenwasserstoffgemisch und deren Unterphase aus Methanol mit steigendem Wassergehalt bestand. Die Mischbarkeit der beiden Phasen nimmt mit steigendem Wassergehalt der Unterphase ab ${ }^{19}$. Die Werte der Trennfaktoren und Verteilungszahlen der Ester sind in Tab. 4. aufgeführt.

Innerhalb der einzelnen Gruppen (gleiche Zusammensetzung der Oberphase) findet man mit steigendem Wassergehalt der Unterphase eine Zunahme des Trennfaktors bzw. eine Abnahme der Zahl der Verteilungsschritte. Der Vergleich der $n$-Werte der einzelnen Gruppen zeigt, daß sich die Mühe des Auf-

19 L. S i e g, Chem. Ing. Techn. 23, 112 [1951]. 
suchens eines geeigneten Lösungsmittelsystems vor Beginn einer Verteilung sicher lohnt, selbst wenn dabei der Trennfaktor nur um wenige Zehnteleinheiten vergrößert wird.

Die in Tab. 4 wiedergegebene Regelmäßigkeit im Gang von Mischbarkeit und Trennfaktor kann zwar als Regel mit Vorteil angewandt werden, sie wird jedoch von Ausnahmen durchbrochen, wie Collan der ${ }^{20}$ in Arbeiten über die Beziehung zwischen chemischer Konstitution und dem Verteilungskoeffizienten in verschiedenen Systemen gezeigt hat. Danach läßt sich das Verteilungsverhalten der verschiedensten Substanzen in verschiedenen Lösungsmittelpaaren gut verstehen, wenn man zu seiner Deutung die Tendenz zur Ausbildung von Wasserstoffbrücken zwischen den Molekülen der gelösten Substanz untereinander und mit der lösenden Phase heranzieht. Auch die Brauchbarkeit von „Schleppersubstanzen“ bei der azeotropen und extraktiven Destillation kann auf dieser Basis qualitativ abgeschätzt werden ${ }^{21}$.

Außer dem Trenn- und dem Volumenfaktor, die sich aus den Verteilungszahlen ergeben, spielt auch die absolute Löslichkeit der Substanzen in den beiden Phasen eine Rolle, da davon die Kapazität eines Lösungsmittelsystems abhängt. Diese ist nach oben hin durch den Gültigkeitsbereich des N e r n s t schen Verteilungssatzes beschränkt. Im Interesse eines möglichst großen Substanzdurchsatzes bei einer Verteilung wird man Lösungsmittelsysteme vermeiden, die diese obere Grenze nicht zu erreichen gestatten.

Außer günstigen Eigenschaften bezüglich der Löslichkeit, Dichte, Oberflächenspannung, Viskosität, leichte Entfernbarkeit nach der Verteilung u.a.m. sind an ein für die Gegenstromverteilung günstiges Phasenpaar also zwei, einander je nach der zu verteilenden Substanz mehr oder weniger ausschließende Anforderungen zu stellen:

1. Möglichst geringe Mischbarkeit der Phasen.

2. Trotzdem genügend große Kapazität des Systems.

Die Schwierigkeit in der Auswahl des richtigen Lösungsmittelpaares besteht also darin, einen großen Trennfaktor und einen nicht zu sehr von 1 verschie-

20 R. C oll a n d e r, Acta chim. scand. 5, 774 [1951]. 21 G. Kortüm u. H. B u ch holz-M e is en he i mer, Die Theorie der Destillation und Extraktion von Flüssigkeiten. Springer Verlag, Berlin/Göttingen/Heidelberg 1952.

22 J. C. S m it h, Ind. Engng. Chem. 41, 2932 [1949].

23 A. W. F r a n c is, Ind. Engng. Chem. 36, 1096 [1944]. denen Volumenfaktor mit einer großen Kapazität des Systems zu vereinigen. Dazu ist die Kenntnis zahlreicher Lösungsmittelsysteme und ihrer Phasendiagramme ${ }^{22}$ sowie die Kenntnis der Löslichkeit der zu verteilenden Substanzen von Nutzen ${ }^{20}$. Da häufig jedoch die Phasendiagramme der in Frage kommenden Systeme nicht gemessen sind, wird man mit Vorteil eine Systematik der Lösungsmittelsysteme zur ersten Orientierung über die Möglichkeiten heranziehen. Eine solche Systematik, die sich auf die gegenseitige Löslichkeit von Flüssigkeiten gründet, ist von F ra ncis $^{23}$, eine ähnliche auch von $\mathrm{Ewell}, \mathrm{H}$ arris on und $\mathrm{B}$ e r g ${ }^{24}$ angegeben worden.

Gelingt es trotz sorgfältiger Vorversuche nicht, ein Lösungsmittelsystem zu finden, in $\operatorname{dem} \beta$ so groß ist, daß die Zahl der zur Verfügung stehenden Verteilungselemente zur Trennung ausreicht, so kann man zu Verfahren übergehen, die eine Gegenstromverteilung über die Zahl der vorhandenen Verteilungselemente hinaus zu größeren Trenneffekten fortzuführen gestatten. Auf die Theorie dieser Entnahmeverfahren und den Vergleich ihrer Trenneffekte untereinander und mit dem Grundprozeß ist früher schon ausführlich eingegangen worden ${ }^{7}$.

$$
\begin{aligned}
& \text { Vergleich des Trenneffektes der } \\
& \text { Gegenstromverteilung mit dem } \\
& \text { anderer Extraktionsverfahren }
\end{aligned}
$$

Die Gegenstromverteilung ist eine Ausführungsform der fraktionierten Extraktion, die durch diskontinuierliche Einstellung des Verteilungsgleichgewichts in einzelnen Verteilungsschritten charakterisiert ist. Im Gegensatz dazu gibt es zahlreiche Extraktionsverfahren, die mit kontinuierlicher Gleichgewichtseinstellung arbeiten, also etwa so, daß in einer Kolonne von unten her leichte Phase und von oben her schwere Phase einander entgegenströmen. Das zu trennende Substanzgemisch wird meist in der Mitte der Kolonne zugeführt (vgl. z. B. ${ }^{25}$ ). Man kann aber auch die schwerere der beiden Phasen stationär machen und von leichter Phase durchströmen lassen. Eine besonders einfache Verwirklichung dieses Prinzips ist die von $\mathrm{K}$ i e s und $\mathrm{D}$ a v i s ${ }^{26}$ angegebene Kaskadenverteilung, während J o h n s o n ${ }^{27}$

24 R. H. Ewell, J. M. H a r r is on u. L. B e r g, Ind. Engng. Chem. 36, 871 [1944].

25 R. R o m et s ch, Helv. chim. Acta 33, 185 [1950].

26 M. W. K i es u. P. L. D a vis, J. biol. Chemistry 189, 637 [1951].

27 J. D. A. J o hn s o n, J. chem. Soc. [London] 1950, 1743 . 
nach dem gleichen Prinzip, aber mit größerem apparativem Aufwand arbeitet.

Eine etwas modifizierte Form der kontinuierlichen, fraktionierten Extraktion, bei der die stationäre Phase durch ein Adsorbens festgelegt wird, ist die Verteilungschromatographie. Hierbei stehen aber häufig außer der Verteilung der Substanzen zwischen den beiden Phasen auch noch Adsorptionskräfte im Spiel, so daß die Verhältnisse theoretisch sehr unübersichtlich werden. Die Trenneffekte dieser kontinuierlichen Extraktionsmethoden können nun mit der Trennwirkung der Gegenstromverteilung auf Grund der Trennfunktion quantitativ verglichen werden.

Für die zahlreichen Ausführungsformen der kontinuierlichen Extraktion (flüssig-flüssig) kann das Verfahren von J o h n s o n ${ }^{27}$ als Repräsentant gelten. In Tab. 5 wird die von J ohnson für sein Verfahren berechnete Anzahl der Trennstufen mit der Zahl der Verteilungselemente verglichen, die für die Gegenstromverteilung (Grundprozeß) zur Erzielung desselben Trenneffektes notwendig sind.

Man erkennt, daß die Gewinnung von Substanzen nach dem Grundprozeß der Gegenstromverteilung wesentlich mehr Verteilungselemente bzw. -schritte erfordert als nach dem kontinuierlich arbeitenden Verfahren von J o hn s on. Für 90-proz. Reinheit ist bei Trennung durch Gegenstromverteilung das 11-fache, für 95-proz. Reinheit das 7,6-fache an Verteilungselementen erforderlich. Nach J o hns on würde bereits eine Verteilungsbatterie mit 100 Elementen zur quantitativen Trennung von Substanzen mit Trennfaktoren bis herab zu $\beta=1,8$ ausreichen, während bei der Gegenstromverteilung dazu mindestens 413 Verteilungselemente erforderlich sind.

Theoretisch sind also die Verfahren mit kontinuierlicher Gleichgewichtseinstellung der Gegenstromverteilung überlegen, jedoch ist zu berücksichtigen, daß der praktischen Durchführung kontinuierlicher Extraktionen große Schwierigkeiten entgegenstehen.

Das Hauptproblem bei den kontinuierlichen Verfahren ist die vollständige und rasche Einstellung des Verteilungsgleichgewichts. Dazu muß die Dispergierung der beiden Phasen so weit getrieben werden, daß man Tröpfchendurchmesser von der Größenordnung $10^{-3} \mathrm{~cm}$ erreicht ${ }^{25}$. Die Beherrschung der Strömungsgeschwindigkeiten der beiden Phasen ist mit

28 L. C. Craig u. A. R. B attersby, J. Amer. chem. Soc. 74, 4019 [1952].

$29 \mathrm{P}$ od bi elniak, Ind. Engng. Chem., Analyt. Ed. 13. 639 [1941].

\begin{tabular}{|c|c|c|c|}
$\begin{array}{c}\% \text { reine } \\
\text { Substanz }\end{array}$ & $\begin{array}{c}\text { Trennfaktor } \\
\beta\end{array}$ & $\begin{array}{c}\text { Trennstufen } \\
\mathrm{t}^{27}\end{array}$ & $\begin{array}{c}\text { Verteilungs- } \\
\text { schritte } n_{\alpha=1}\end{array}$ \\
\hline 90 & 1,6 & 30 & 331 \\
90 & 1,5 & 40 & 445 \\
90 & 1,4 & 60 & 646 \\
95 & 1,6 & 50 & 389 \\
95 & 1,5 & 68 & 523 \\
95 & 1,4 & 100 & 760 \\
\hline
\end{tabular}

$t=$ Trennstufen nach Johnson ${ }^{2 \tau}, n_{a^{*}=1}$ Verteilungsschritte für den Grundprozeß der Gegenstromverteilung bei $a=1$.

Tab. 5 .

technischem Aufwand verknüpft, und die Wirksamkeit einer solchen Extraktion ist in hohem $\mathrm{Ma}$ von den Eigenschaften der beiden Phasen abhängig, so daß eine für ein System brauchbare Kolonne nicht notwendig auch für ein anderes System geeignet sein muß. Die Methode wird deshalb zwar in der Technik zur präparativen Trennung von Substanzgemischen häufig eingesetzt, hat sich aber im Laboratorium trotz mancher Ansätze bisher nicht eingeführt. Dagegen hat sich eine Verteilungsbatterie zur Gegenstromverteilung, mit der mehr als 2000 Verteilungsschritte nach dem Grundprozeß ausgeführt werden können, schon seit längerem bewährt ${ }^{28}$.

Im Gegensatz dazu ist die Verteilungschromatographie durch die Arbeiten von Martin und S yng e sehr rasch im Labor eingeführt worden. Besonders in der Ausführungsform der Papierchromatographie hat sie sich zur qualitativen Erkennung kleinster Substanzmengen sehr gut bewährt.

Die Wirksamkeit einer Säule zur Verteilungschromatographie wird meist durch die Zahl der theoretischen Böden ausgedrückt, wie es für Destillationsund kontinuierlich arbeitende Extraktionskolonnen üblich ist. Man ermittelt also den kleinsten Abschnitt einer Säule, in der sich die beiden Phasen bestimmter Zusammensetzung vollständig ins Gleichgewicht setzen. Dieser kleinste Abschnitt ist dann der Wirkung eines theoretischen Bodens äquivalent. Bei den besten Destillationskolonnen ${ }^{29}$ und Extraktionskolonnen ${ }^{30}$ entspricht etwa $1 \mathrm{~cm}$ Säulenhöhe einem theoretischen Boden. Demgegenüber sind die Säulen zur Verteilungschromatographie wesentlich wirksamer. $\mathrm{M}$ artin und Synge $\mathrm{e}^{15}$ haben gefunden, daß bei der von ihnen verwendeten Säule $0,002 \mathrm{~cm}$ einem theoretischen Boden äquivalent sind.

30 Ney u. L ochte, Ind. Engng. Chem. 33, 825 [1941]. 
Bei der Gegenstromverteilung (Grundprozeß) entspricht ein Verteilungsschritt der Wirkung eines theoretischen Bodens. Man kann also anstatt von einer Verteilungsbatterie mit $z$ Elementen auch von einer Batterie mit $n=z-1$ theoretischen Böden sprechen und die Trennfunktion zum quantitativen Vergleich der Trennwirkung von Gegenstromverteilung und Verteilungschromatographie heranziehen. Dabei ist jedoch zu beachten, daß die gleiche Anzahl theoretischer Böden bei zwei verschiedenen Trennungsverfahren einander in der Trennwirkung nicht äquivalent $\mathrm{zu}$ sein brauchen. Vielmehr existiert für jedes Verfahren eine spezielle Trennfunktion, in die die Breite der Verteilungskurven eingeht. Diese ist für die Verteilungskurven jedes Verfahrens spezifisch.

Nach dem Befund von M a r tin und S y n ge entspricht eine $2 \mathrm{~cm}$ lange Säule zur Verteilungschromatographie der Wirkung von 1000 theoretischen Böden. Die gleiche Anzahl theoretischer Böden ist mit der Gegenstromverteilung nur mit einem relativ großen apparativen Aufwand zu erreichen. So hat $\mathrm{M}$ a r t in ${ }^{51}$ an einer $20 \mathrm{~cm}$ langen Säule ein Gemisch von Palmitin- und Myristinsäure quantitativ getrennt, wozu $\mathrm{Craig}{ }^{52}$ eine Gegenstromverteilung mit 400 Verteilungsschritten benötigte. In anderen Fällen ist jedoch die Zahl der theoretischen Böden einer Chromatographiesäule wesentlich kleiner und kann bei derselben Säule von Substanz zu Substanz beträchtlich schwanken. Diese schlechte Reproduzierbarkeit der Trennwirkung einer Säule zeigt, daß die Annahme reiner Verteilungsvorgänge bei diesen Säulen sehr stark idealisiert ist. C r a i g ist deshalb der Meinung, daß zwischen Verteilungs- und Adsorptionschromatographie mehr Verwandtschaft besteht als zwischen Verteilungschromatographie und Gegenstromverteilung. Dabei darf jedoch nicht übersehen werden, daß die Gegenstromverteilung zwar unter idealen „Verteilungsbedingungen“, aber nur bei großem apparativem Aufwand mit demselben Trenneffekt wie die Verteilungschromatographie arbeitet, wie zahlreiche Beispiele aus der Praxis zeigen ${ }^{32,33}$ Ähnliches gilt, sinngemäß übertragen, auch für die Tswettsche Adsorptionschromatographie.

21 A. J. P. Martin u. C. A. How ard, Biochem. J. 44, 532 [1950].

32 L. C. C r a i g, Analyt. Chemistry 22, 1346 [1950].

33 S. M o ore u. W. H. Stein, J. biol. Chemistry 178, 53 [1949].
Die Erkenntnis, daß die Trennwirkung der Gegenstromverteilung nur bei Anwendung von sehr vielen Verteilungsschritten in die gleiche Größenordnung, wie die einer Säule zur Verteilungschromatographie kommt, vermindert den Wert der Methode in keiner Weise. Man wird vielmehr zur Lösung schwieriger Trennungsprobleme die Nachteile von Adsorptionsund Verteilungs- bzw. Papierchromatographie und Gegenstromverteilung durch zweckmäßige Anwendung der Methoden auszugleichen versuchen.

Bei zahlreichen Arbeiten am hiesigen Institut haben wir gefunden, daß sich Gegenstromverteilung und Adsorptionschromatographie in ausgezeichneter Weise ergänzen. Häufig gelang die Reindarstellung von Substanzen, die durch Adsorptionschromatographie nicht zu trennen waren, durch Gegenstromverteilung mit wenigen Verteilungsschritten, und auch der umgekehrte Fall wurde häufig beobachtet. Ähnliches gilt für das Verhältnis von Verteilungschromatographie und Gegenstromverteilung. Zwar ist hier der Trenneffekt bei der Verteilungschromatographie meist größer als bei den gewöhnlichen Verteilungsbatterien, jedoch können bei der Gegenstromverteilung wesentlich größere Mengen präparativ bearbeitet werden.

Die gute, gegenseitige Ergänzung von Gegenstromverteilung und Adsorptionschromatographie kann vielleicht zur Beantwortung der Frage herangezogen werden, warum man bei der Verteilungschromatographie in relativ kurzen Säulen eine so außerordentlich große Anzahl von theoretischen Böden erzielt. Man könnte annehmen, daß dies auf die gleichzeitige Anwendung des Adsorptions- und des Verteilungsprinzips und Additivität der Effekte zurückzuführen ist.

Durch den Vergleich der Trenneffekte sowie der Vor- und Nachteile von Gegenstromverteilung, Adsorptions- und Verteilungschromatographie wurde zu zeigen versucht, daß es zur Lösung schwieriger Trennungsprobleme zwar die Methode schlechthin nicht gibt, daß aber die sinnvolle Kombination mehrerer Methoden ein Optimum zu leisten vermag.

Herrn Prof. Dr. A. Bu te n a nd t möchte ich für die Förderung und Unterstützung der Arbeit besonderen Dank aussprechen. Für wertvolle Diskussionen und Anregungen bin ich den Herren Dr. P eter Karls on, Max-Planck-Institut für Biochemie, und Herrn Studienassessor $\mathrm{Helmut} \mathrm{Ri}$ ekert, Mathematisches Institut der Universität Tübingen, zu Dank verbunden. 